\title{
The Use of ANFIS and RBF to Model and Predict the Inhibitory Concentration Values Determined by MTT Assay on Cancer Cell Lines
}

\author{
A. Rezaei \\ Electrical Engineering Department, Kermanshah University of Technology, Kermanshah, Iran \\ E-mail: unrezaei@yahoo.com \\ L. Noori, M. Taghipour \\ Electrical Engineering Department, Kermanshah University of Technology, Kermanshah, Iran \\ Department of Biomedical Engineering, Kermanshah University of Medical Sciences, Kermanshah, Iran \\ E-mail: Leila_noori62@yahoo.com, mostafa.taghipour@kums.ac.ir
}

\begin{abstract}
The computational intelligence such as artificial neural network (ANN) and fuzzy inference system (FIS) is a strong tool for prediction and simulation in engineering applications. In this paper, radial basis function (RBF) network and adaptive neuro-fuzzy inference system (ANFIS) are used for prediction of $\mathrm{IC}_{50}$ (the 50\% inhibitory concentration) values evaluated by the MTT assay in human cancer cell lines. For developing of the proposed models, the input parameters are the concentration of the drug and the types of cell lines and the output is $\mathrm{IC}_{50}$ values in the A549, H157, $\mathrm{H} 460$ and $\mathrm{H} 1975$ cell lines. The predicted $\mathrm{IC}_{50}$ values using the proposed RBF and ANFIS models are compared with the experimental data. The obtained results show that both RBF and ANFIS models have achieved good agreement with the experimental data. Therefore, the proposed RBF and ANFIS models are useful, reliable, fast and cheap tools to predict the $\mathrm{IC}_{50}$ values determined by the MTT assay in human cancer cell lines.
\end{abstract}

Index Terms - Computational intelligence, Radial basis function, Adaptive neuro-fuzzy inference system, Lung cancer, MTT assay.

\section{INTRODUCTION}

Lung cancer is leading cause of cancer-related deaths among cancer patients [1], with an estimated of 1.61 million new cases and 1.38 million deaths worldwide in 2008 [2]. Lung cancer has two main types, non-small cell lung cancer (NSCLC) and small cell lung cancer (SCLC). NSCLC accounts for approximately $85 \%$ of all lung cancer cases and making it a major global public health concern [1]. Doxorubicin (DOX, trade name Adriamycin) is a naturally occurring anthracycline antibiotic [3]. DOX has been in use since the late 1960s and is a widely used anti-cancer agent in chemotherapy [4].

DOX, alone, or in combination, is an important drug in the treatment of the lung cancer and other cancers, but bone marrow and cardio-toxic effects of DOX limit its use in clinical medicine [5]. Therefore, there is a need to explore the adjuvants and/or natural compounds that can effectively reduce the doxorubicin-induced toxicity to improve its chemotherapeutic efficacy [6]. A number of adjuvants and natural compounds have been tested to increase DOX chemo-efficiency [5]. Chrysin is one of them. Chrysin (5,7-dihydroxyflavone) is a naturally occurring flavonoid that is widely distributed in medicinal herbs [5] and has displayed numerous chemopreventive properties against the chemically induced toxicities.

Chrysin has also shown to have the anti-growth properties against the various cancers in animal models and in the different human cancer cell lines like human colorectal cell line and prostate cancer cell line [6,7]. Recently reported study by Brechbuhl et al. [8], showed that Chrysin induces cancer cell death synergistically in combination with DOX $[6,8]$. In the study of Brechbuhl et al. [8], MTT [3-(4,5-dimethylthiazol-2-yl)-2-,-5diphenyltetrazolium bromide] assay has been conducted to measure the cytotoxicities ( $\mathrm{IC}_{50}$ values) of the combination drug therapy with Chrysin and DOX against the cancer cell lines. The MTT colorimetric assay is one of the most widely used assays for estimating the cytotoxicity, viability of animal or bacterial cells and proliferation studies in the cell biology $[9,10]$.

This method has been widely used to screen for the cytotoxic compounds during the development of chemotherapy or to screen for the unwanted cytotoxic effects during the preclinical studies [10].

By considering the substantial failure rate of adjuvants and/or drug candidates in the late stage development and the expensive and time consuming process of measuring toxic effects, the predictive tools that can eliminate inappropriate compounds seems necessary [11] and can play significant role in providing the missing data of cytotoxicity of new adjuvants and/or drug candidates.

In the recent decades, new methods such as artificial neural networks (ANNs) and adaptive neuro-fuzzy inference system (ANFIS) have been used to develop the 
predictive models to estimate the needed parameters. Computational intelligence $(\mathrm{CI})$ techniques are now being used as an alternative to the statistical tools [12-15].

In this paper, the applicability of ANFIS and radial basis function (RBF) network, which is a type of ANNs, for prediction of $\mathrm{IC}_{50}$ values evaluated by MTT assay in human cancer cell lines are investigated. For this purpose, we are used the experimental data reported in Brechbuhl et al. [8] for developing the proposed models.

The prediction capability of the proposed ANFIS and RBF models can be used to fill the gaps of data for untested concentrations and their $\mathrm{IC}_{50}$ values of new adjuvants and/or drug candidates for cancer drug screening. These models can be used to predict the unknown data by saving time and money.

\section{EXPERIMENTAL DATA}

For developing the proposed ANFIS and RBF models, we used the experimental data. To obtain the experimental data, the $\mathrm{IC}_{50}$ of combination drug therapy with Chrysin and DOX has been determined against the cell lines by the MTT assay.

Briefly, the lung non-small cell epithelial cancer (NSCLC) cell lines A549, H157, H460 and H1975 have been cultured at $37^{\circ} \mathrm{C}$ with $5 \% \quad \mathrm{CO}_{2}$. The NSCLC cell lines have been grown in the media and supplements and then have been seeded in 96-well plates.

After indicated treatments, $20 \mu \mathrm{l} /$ well of MTT solution has been added and incubated for 3-4 h, then $150 \mu \mathrm{l} /$ well of DMSO has been added to dissolve formazan crystals. The absorbance of each well has been observed by a plate reader at a test wavelength of $570 \mathrm{~nm}$. The $\mathrm{IC}_{50}$ values have been calculated using prism version 5. For more details of the assay conditions and the experimental data, the readers are advised to refer to the work of Brechbuhl et al. [8].

\section{Modeling UsING RBF}

In the mathematical modeling a radial basis function (RBF) network, is an ANN, which uses RBFs as the activation functions. Its output is a linear combination of RBFs of the inputs and the parameters of neurons. RBF networks have many applications such as function approximation, prediction of time series, and system control.

The RBF has a feed forward ANN structure consists of three layers (one input layer, one hidden layer and one output layer) as shown in Fig.1. [16, 17]. The input layer is made up of the source nodes that connect the network to its environment.

The second layer consists of a set basis function units that carry out a nonlinear transformation from the input space to the hidden space [18]. The output from $\mathrm{j}^{\text {th }}$ neuron of the hidden layer is given by:

$$
Z_{j}=K\left(\frac{\left\|X-\mu_{j}\right\|}{\sigma_{j}^{2}}\right) j=1,2, \ldots, k
$$

where $\mathrm{k}$ is the number of neurons in the hidden layer, $\sigma_{j}$ is the width of the receptive field in the input space from unit $\mathrm{j}, K$ is a strictly positive radially symmetric function (kernel) with a unique maximum at its center $\left(\mu_{j}\right)$, which drops off rapidly to zero away from the center. This implies that $Z_{j}$ has an appreciable value only when the distance $\left\|X-\mu_{j}\right\|$ is smaller than the width $\sigma_{j}$ [19]. Given an input vector $X$, the output of the $m^{\text {th }}$ neuron in the output layer is given by:

$$
y_{m}(x)=\sum_{j=1}^{k} w_{j m} z_{j}(x) \quad m=1,2, \ldots, M
$$

where $w_{j m}$ is the weighting factor. The training process of the RBF networks can be typically done by a two-step algorithm. The center vectors of the RBF functions in the hidden layer are chosen randomly from some set of examples in the first step.

Also, these center vectors can be determined by kmeans clustering. In the second step, a linear model is fitted to the hidden layer's outputs using the least squares function.

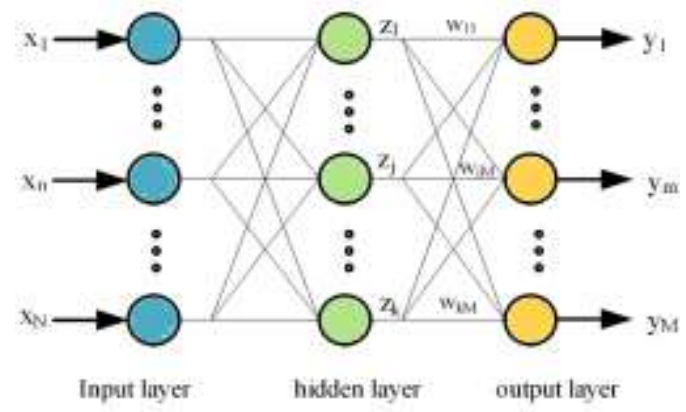

Fig.1. RBF structure.

The RBF network is a strong tool for prediction and simulation in engineering applications. In this study, the $\mathrm{RBF}$ model is developed for prediction of the inhibitory concentration values assessed by the MTT assay on human lung epithelial cancer cell lines co-treated with Chrysin and doxorubicin. The inputs of the proposed $\mathrm{RBF}$ model are the concentrations and the types of the cell lines and the output is the $\mathrm{IC}_{50}$ value in the $\mathrm{A} 549$, H157, H460 and H1975 cell lines. The data set required for training the proposed RBF model is obtained using the experimental values [8]. To build the RBF model, the experimental data are divided into two sets. The number of the samples for training and testing are chosen 21 (75\%) and 7 (25\%), respectively. For training the proposed RBF model, a computer program is developed using MATLAB 7.0.4 software. The best proposed RBF network being used in this study is obtained with 21 neurons in the hidden layer. 


\section{MODELING USING ANFIS}

An adaptive neuro-fuzzy inference system (ANFIS) is a kind of artificial neural network (ANN) that is based on Takagi-Sugeno fuzzy inference system (FIS). ANFIS is a universal estimator. ANFIS was developed in order to integrate the benefits of both ANNs and FISs in a single framework to approximate nonlinear functions. ANFIS is a multilayer feed-forward network, which combines the advantages of both ANN (e.g., learning capacities, optimization capacities, and connectionist structures) and FIS (e.g., human like "IF-THEN" rules, thinking and ease of incorporating expert knowledge) to map an input space to an output space $[20,21]$. In this study, the prediction of the inhibitory concentration values assessed by the MTT assay on the human lung epithelial cancer cell lines cotreated with Chrysin and doxorubicin using ANFIS is presented. The proposed ANFIS model is shown in Fig.2., where the inputs are the concentrations and the types of the cell lines and the output is $\mathrm{IC}_{50}$ value in the A549, H157, H460 and H1975 cell lines.

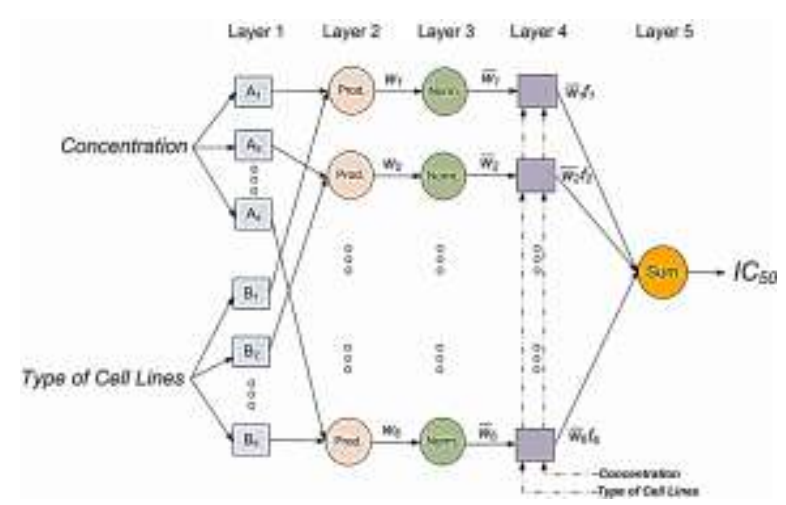

Fig.2. The proposed ANFIS model.

A description of the layers in the proposed ANFIS model is defined as follows [22, 23]:

Layer 1. Each node in this layer is an adjustable node, marked by the square node. The node functions of this layer are given by:

$$
\begin{aligned}
& A_{i}(\text { Con })=\mu_{A i}(\text { Con }), \quad i=1,2, \ldots, 6 \\
& B_{i}(T C L)=\mu_{B i}(T C L), \quad i=1,2, \ldots, 6
\end{aligned}
$$

where Con (concentration) and TCL (type of cell lines) are the inputs of the model and $\mu_{A}(\mathrm{Con})$ and $\mu_{B}$ (TCL) are the fuzzy membership functions. In this study the membership function is Gaussian membership function and is given by:

$$
G(x)=\operatorname{Exp}\left(-\frac{0.5\left(x-a_{i}\right)^{2}}{b_{i}^{2}}\right)
$$

Parameters in this layer are referred to as premise parameters (nonlinear parameters). As the values of these parameters change, the Gaussian function varies accordingly.

Layer 2. Each node in this layer is an adjustable node, marked by the circle node. The output of this layer is given by:

$$
W_{i}=A_{i}(\text { Con }) \times B_{i}(T C L), \quad i=1,2, \ldots, 6
$$

Layer 3. Each node in this layer is a fixed node, marked by the circle node. This layer estimates the ratio of the $i^{\text {th }}$ rule's firing strengths to the sum of all rule's firing strengths as follows:

$$
\bar{W}_{i}=\frac{W_{i}}{\sum_{j=1}^{6} W_{j}}, \quad i=1,2, \ldots, 6
$$

Layer 4. Each node in this layer is an adjustable node, marked by the square node. The output of this layer is given by:

$$
f_{i}=\bar{W}_{i}\left(m_{i} \operatorname{Con}+n_{i} T C L+k_{i}\right) \quad, i=1,2, \ldots, 6
$$

Where $m_{i}, n_{i}$ and $k_{i}$ are called the consequent parameters (linear parameters).

Layer 5. Each node in this layer is a fixed node, marked by the circle node. The output of this layer is given by:

$$
I C_{50}=\sum_{i=1}^{6} f_{i}
$$

The aim of the ANFIS training is to tune all the parameters (the consequence and premise parameters) to make the ANFIS output match the training data. ANFIS uses the Hybrid learning algorithm, which is a two-step process. In the first step (the forward direction), the consequent parameters are identified by the least square estimation and using the gradient descent the premise parameters are updated. In the second step (the backward direction), the premise parameters are updated by the feedbacked error values. When all the values of the parameters are changed, the membership functions are also modified. The data set required for training the proposed ANFIS network is obtained using the experimental values. For developing the ANFIS model, the experimental data are divided into two sets. The number of samples for training and testing are $21(75 \%)$ and $7(25 \%)$, respectively. In this study, different ANFIS structures are tested and optimized to obtain the best ANFIS configuration. By testing the various ANFIS structures with the different number of membership functions, we obtained the optimal structure with 6 membership functions for each input. Also, we tested all types of the membership functions like Bell, Sigmoid, Triangle, Trapezoid and Gaussian. The Gaussian membership function in comparison with the others had the better results. Table 1 . shows the specification of the best proposed ANFIS model. 
Table 1. Specification of the proposed ANFIS model.

\begin{tabular}{|l|l|}
\hline Type & Sugeno \\
\hline Inputs/outputs & $2 / 1$ \\
\hline No. of input membership functions & 6 for each input \\
\hline No. of output membership functions & 6 \\
\hline Input membership function type & Gaussian \\
\hline Output membership function type & linear \\
\hline No. of fuzzy rules & 6 \\
\hline No. of nonlinear parameters & 48 \\
\hline No. of linear parameters & 24 \\
\hline No. of epochs & 150 \\
\hline
\end{tabular}

\section{RESULTS AND DISCUSSIONS}

In this section, the proposed RBF and ANFIS models for the prediction of the $\mathrm{IC}_{50}$ values are compared with each other and with the experimental data. The training and testing results for the proposed RBF and ANFIS models in comparison with the experimental data are shown in Table 2., Table 3., Fig.3. and Fig.4., where the mean relative error percentage (MRE \%), the mean absolute error (MAE), the root mean square error (RMSE), and the correlation factor (CF) are calculated by the following equations:

$$
\begin{aligned}
& M R E \%=100 \times \frac{1}{N} \sum_{i=1}^{N}\left|\frac{X_{i}(E x p)-X_{i}(\operatorname{Pr} e d)}{X_{i}(\operatorname{Exp})}\right| \\
& M A E=\frac{1}{N} \sum_{i=1}^{N}\left|X_{i}(\operatorname{Exp})-X_{i}(\operatorname{Pr} e d)\right|
\end{aligned}
$$

$$
\begin{gathered}
R M S E=\left[\frac{\sum_{i=1}^{N}\left(X_{i}(\text { Exp })-X_{i}(\operatorname{Pr} e d)\right)^{2}}{N}\right]^{0.5} \\
C F=1-\left[\frac{\sum_{i=1}^{N}\left(X_{i}(\operatorname{Exp})-X_{i}(\operatorname{Pr} e d)\right)^{2}}{\sum_{i=1}^{N}\left(X_{i}(\operatorname{Exp})\right)^{2}}\right]
\end{gathered}
$$

where ' $X($ Exp $)$ ' and ' $X($ Pred $)$ ' stand for the experimental and predicted (RBF or ANFIS) values, respectively and $\mathrm{N}$ is the number of data. Table 2. shows the comparison between the experimental and predicted (RBF and ANFIS) results for training data. While in Table 3. the comparison between the experimental and predicted (RBF and ANFIS) results for testing data is shown. As it can be seen in Table 2. and Table 3., there is a remarkable agreement between the experimental and predicted values using both proposed ANFIS and RBF models, which show that the proposed ANFIS and RBF models can be used as the accurate and reliable tools to predict the $\mathrm{IC}_{50}$ values. From Table 2., Table 3., Fig.3. and Fig.4. it is clear that both ANFIS and RBF models have good agreement with the experimental, also the proposed ANFIS model is more accurate than the proposed RBF model. The ANFIS model could significantly reduce the overall (training and testing) MRE\% to less than $0.95 \%$ in comparison with the RBF model, which has the overall (training and testing) MRE\% less than 4.5\%. Also, the ANFIS model has a number of epochs 150, which is needed to reach the convergence. In comparison with the proposed RBF model, the time needed for the training the ANFIS model was less than the required time to design the proposed RBF model.

Table 2. Comparison between the experimental and predicted (RBF and ANFIS) results for training data.

\begin{tabular}{|c|c|c|c|c|}
\hline \multirow{2}{*}{ Type of cell lines } & \multirow{2}{*}{ Chrysin $(\mu \mathrm{M})$} & \multicolumn{3}{|c|}{$\mathrm{IC}_{50}$} \\
\cline { 3 - 5 } & & Experimental & $\mathrm{RBF}$ & ANFIS \\
\hline A549 & 0 & 0.365 & 0.3542 & 0.3651 \\
\hline A549 & 5 & 0.291 & 0.2632 & 0.2907 \\
\hline A549 & 10 & 0.264 & 0.2446 & 0.2645 \\
\hline A549 & 20 & 0.212 & 0.1933 & 0.2121 \\
\hline A549 & 25 & 0.223 & 0.2213 & 0.2228 \\
\hline A549 & 30 & 0.238 & 0.2397 & 0.2376 \\
\hline H157 & 5 & 0.367 & 0.3485 & 0.3671 \\
\hline H157 & 10 & 0.35 & 0.3595 & 0.3520 \\
\hline H157 & 15 & 0.317 & 0.3199 & 0.3169 \\
\hline H157 & 25 & 0.325 & 0.3523 & 0.3248 \\
\hline H157 & 30 & 0.351 & 0.0669 & 0.3519 \\
\hline H460 & 0 & 0.067 & 0.0358 & 0.0670 \\
\hline H460 & 10 & 0.035 & 0.0285 & 0.0350 \\
\hline H460 & 15 & 0.03 & 0.0329 & 0.0298 \\
\hline H460 & 20 & 0.033 & 0.0167 & 0.0329 \\
\hline H460 & 30 & 0.015 & 0.0649 & 0.0148 \\
\hline H1975 & 0 & 0.066 & 0.0438 & 0.0660 \\
\hline H1975 & 5 & 0.042 & 0.0275 & 0.0418 \\
\hline H1975 & 15 & 0.028 & 0.035 & 0.0287 \\
\hline H1975 & 20 & 0.034 & 0.0337 & 0.0330 \\
\hline H1975 & 30 & 0.035 & 0.0346 \\
\hline
\end{tabular}


Table 3. Comparison between the experimental and predicted (RBF and ANFIS) results for testing data.

\begin{tabular}{|c|c|c|c|c|}
\hline \multirow{2}{*}{ Type of cell lines } & \multirow{2}{*}{ Chrysin $(\mu \mathrm{M})$} & \multicolumn{3}{|c|}{ IC $_{50}$} \\
\cline { 3 - 5 } & & Experimental & RBF & ANFIS \\
\hline A549 & 15 & 0.21 & 0.2294 & 0.2059 \\
\hline H157 & 0 & 0.589 & 0.5652 & 0.5878 \\
\hline H157 & 20 & 0.351 & 0.3413 & 0.3525 \\
\hline H460 & 5 & 0.033 & 0.0347 & 0.0335 \\
\hline H460 & 25 & 0.016 & 0.0173 & 0.0162 \\
\hline H1975 & 10 & 0.042 & 0.0435 & 0.0416 \\
\hline H1975 & 25 & 0.034 & 0.0321 & 0.0350 \\
\hline
\end{tabular}
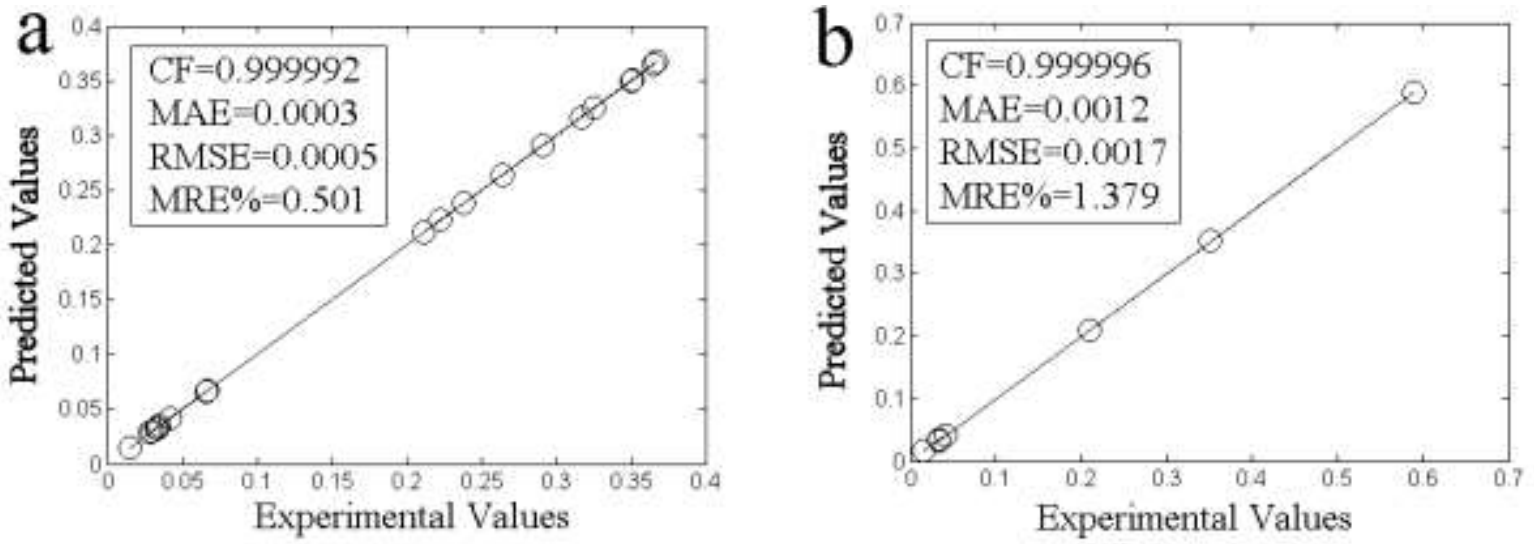

Fig.3. Comparison of the proposed ANFIS model with the experimental for (a):training data and (b):testing data.
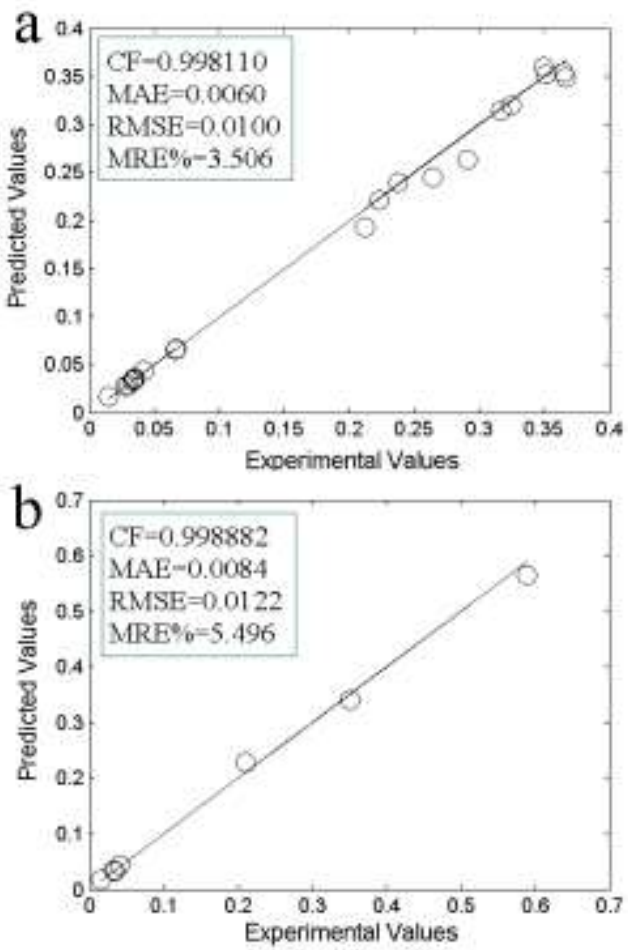

Fig.4. Comparison of the proposed RBF model with the experimental for (a):training data and (b):testing data.
In Fig.5. and Fig.6. the obtained results for $\mathrm{IC}_{50}$ using the proposed ANFIS model (solid line) and the experimental (circle mark) versus concentration of Chrysin (in the range of $0 \mu \mathrm{M}$ to $30 \mu \mathrm{M}$ ) for the various type of cell lines (A549, H157, H460 and H1975) are shown. Both predicted values and experimental data indicate that by increasing the Chrysin from 0 to $30 \mathrm{uM}$ (for the type of cell lines A549), the $\mathrm{IC}_{50}$ sharply decreases, while with further increasing the Chrysin, the $\mathrm{IC}_{50}$ increases. In this case, the minimum $\mathrm{IC}_{50}(0.213)$ is happened in Chrysin=16.5 $\mu \mathrm{M}$. For the cell lines type $\mathrm{H} 157$, by increasing the Chrysin from 0 to $30 \mathrm{uM}$ the $\mathrm{IC}_{50}$ sharply decreases at first, while further increasing the Chrysin has no significant effect on the $\mathrm{IC}_{50}$. In this case, the minimum $\mathrm{IC}_{50}(0.32)$ is happened in Chrysin $=15 \mu \mathrm{M}$. For all types of cell lines, the maximum $\mathrm{IC}_{50}$ occurs at Chrysin=0. From Fig.5. and Fig.6. the maximum $\mathrm{IC}_{50}$ (0.5878) was happened in Chrysin $=0 \mu \mathrm{M}$ for type of cell lines H157. Also the minimum $\mathrm{IC}_{50}$ (0.0140) was happened in Chrysin=28 $\mu \mathrm{M}$ a for type of cell lines $\mathrm{H} 460$. From these results it is clear that the proposed RBF and ANFIS models can be used as the accurate models for prediction of the inhibitory concentration values accessed via the MTT assay on human lung epithelial cancer cell lines co-treated with Chrysin and doxorubicin. 

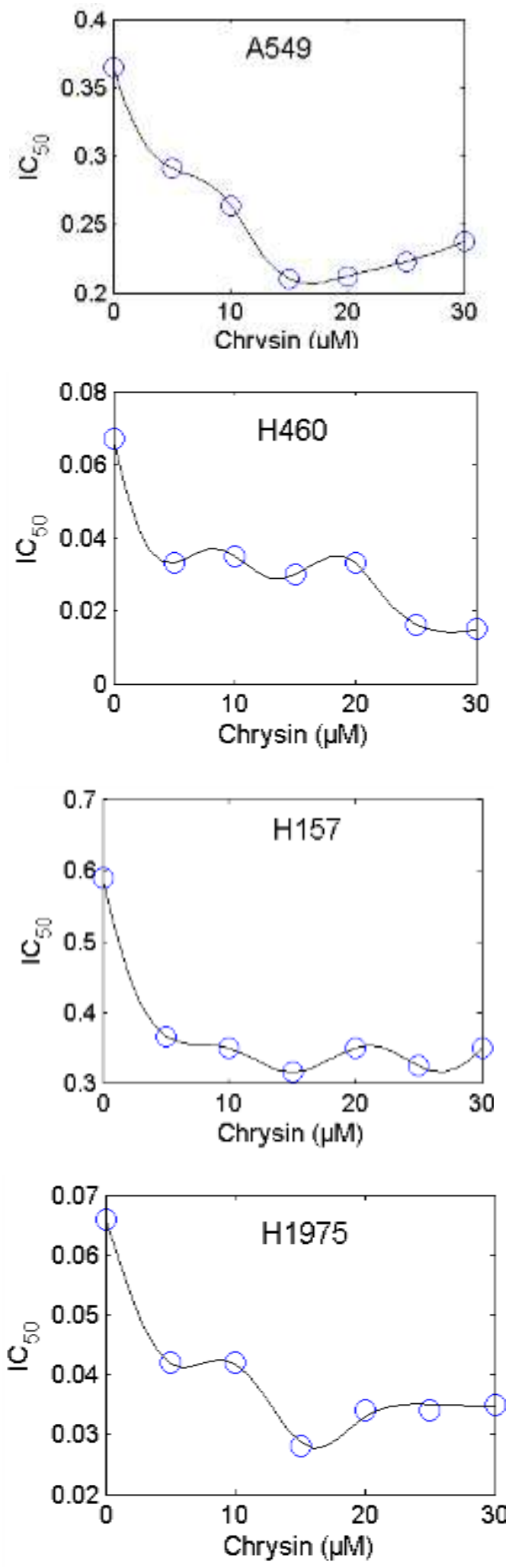

Fig.5. Comparison between the experimental (circle mark) and predicted results using the proposed ANFIS model (solid line) for the types of cell lines A549, H157, H460 and H1975.

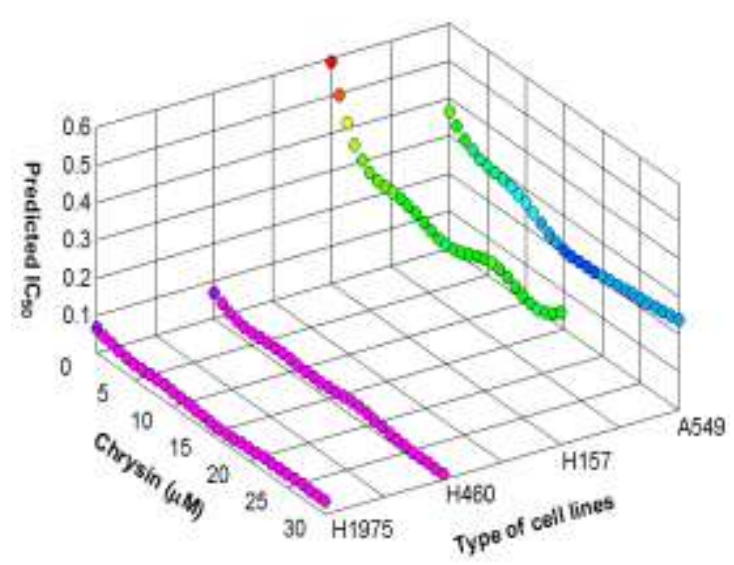

Fig.6. The obtained $\mathrm{IC}_{50}$ using the proposed ANFIS model as a function of Chrysin in A549, H157, H460 and H1975 cells.

\section{CONCLUSIONS}

The purpose of this study was to examine the applicability of the RBF and ANFIS models for prediction of the $\mathrm{IC}_{50}$ values evaluated by the MTT assay in the human lung cancer cell lines. The comparison showed that these models are efficient tools for filling the gaps of data for untested concentrations and their $\mathrm{IC}_{50}$ values of new adjuvants and/or drug candidates with saving time and money. The results of the present study can be shown as follow:

(a) Comparison of the predicted (The RBF and ANFIS models) and experimentally evaluated $\mathrm{IC}_{50}$ values shows that there is a good agreement between them.

(b) The proposed ANFIS model is more accurate than the proposed RBF model.

Therefore, the RBF and ANFIS models can be used as the fast and cheap tools for prediction of the $\mathrm{IC}_{50}$ values with an acceptable accuracy in situations, where the urgency and/or lack of money prevent a thorough cytotoxicity assay like MTT from being conducted.

\section{REFERENCES}

[1] C.J. Langer, T. Mok, P.E. Postmus, "Targeted agents in the third-/fourth-line treatment of patients with advanced (stage III/IV) non-small cell lung cancer (NSCLC)", Cancer Treatment Reviews, Vol. 39, pp. 252-260, 2013.

[2] D. Chen, Y. Zhang, X. Zhang, J. Li, B. Han, S. Liu, L. Wang, Y. Ling, S. Mao, X. Wang, "Overexpression of integrin-linked kinase correlates with malignant phenotype in non-small cell lung cancer and promotes lung cancer cell invasion and migration via regulating epithelial-mesenchymal transition (EMT)-related genes", Acta Histochemica ,Vol. 115, pp.128-136, 2013. 
[3] S. AbuHammad, M. Zihlif, "Gene expression alterations in doxorubicin resistant MCF7 breast cancer cell line", Genomics, Vol. 101, pp. 213-220, 2013.

[4] F. Hellmers, P. Ferguson, J. Koropatnick, R. Krull, A. Margaritis, "Characterization and in vitro Cytotoxicity of Doxorubicin-loaded- $\gamma$-Polyglutamic Acid-Chitosan Composite Nanoparticles," Biochemical Engineering Journal , 2013.

[5] J.J. Shao, A.P. Zhang, W. Qin, L. Zheng, Y.F. Zhu, X. Chen, "AMP-activated protein kinase (AMPK) activation is involved in chrysin-induced growth inhibition and apoptosis in cultured A549 lung cancer cells," Biochemical and Biophysical Research Communications , Vol. 423, pp. 448-453, 2012.

[6] R. Khan, A.Q. Khan, W. Qamar, A. Lateef, M. Tahir, M.U. Rehman, F. Ali, S. Sultana, "Chrysin protects against cisplatin-induced colon. toxicity via amelioration of oxidative stress and apoptosis: Probable role of p38MAPK and p53", Toxicology and Applied Pharmacology, Vol. 258, pp. 315-329, 2012.

[7] M.U. Rehman, M. Tahir, A.Q. Khan, R. Khan, A. Lateef, O. Hamiza, W. Qamar, F. Ali, S. Sultana, "Chrysin suppresses renal carcinogenesis via amelioration of hyperproliferation," oxidative stress and inflammation: Plausible role of $N F-\kappa B$. Toxicology Letters, Vol. 216, pp. 146-158, 2013.

[8] H.M. Brechbuhl, R. Kachadourian, E. Min, D. Chan, B.J. Day, "Chrysin enhances doxorubicin-induced cytotoxicity in human lung epithelial cancer cell lines: The role of glutathione", Toxicology and Applied Pharmacology, Vol. 258, pp. 1-9, 2012.

[9] H. Wang, F. Wang, X. Tao, H. Cheng, "Ammoniacontaining dimethyl sulfoxide: An improved solvent for the dissolution of formazan crystals in the 3-(4,5dimethylthiazol-2-yl)-2,5-diphenyl tetrazolium bromide (MTT) assay", Analytical Biochemistry, Vol. 421, pp. 324-326, 2012.

[10] Y. Li, W. Huang, S. Huang, J. Du, C. Huang, "Screening of anti-cancer agent using zebrafish: Comparison with the MTT assay", Biochemical and Biophysical Research Communications, Vol. 422, pp. 85-90, 2012.

[11] O. Deeb, "Correlation ranking and stepwise regression procedures in principal components artificial neural networks modeling with application to predict toxic activity and human serum albumin binding affinity", Chemometrics and Intelligent Laboratory Systems, Vol. 104, pp. 181-194, 2010.

[12] I. Yilmaz, O. Kaynar, "Multiple regression, ANN (RBF, MLP) and ANFIS models for prediction of swell potential of clayey soils. Expert Systems with Applications," Vol. 38, pp. 5958-5966, 2011.

[13] R. Vern, S.K. Dubey, "Evaluating the Maintainability of a Software System by using Fuzzy Logic Approach," IJITCS, Vol. 7, pp. 67-72, 2014

[14] T.S. Hai, L.H. Thai, N.T. Thuy, "Facial Expression Classification Using Artificial Neural Network and KNearest Neighbor", IJITCS, Vol. 7, pp. 27-32, 2015.

[15] S. Datta, J. Paulchoudhury, "A Comparative Study on the Performance of Fuzzy Rule Base and Artificial Neural Network towards Classification of Yeast Data," IJITCS, Vol. 7, pp. 40-47, 2015.

[16] S. Haykin, "Neural Networks: A Comprehensive Foundation," Prentice-Hall, 1999.

[17] A. Shahsavand, A. Ahmadpour, "Application of Optimal Rbf Neural Networks for Optimization and Characterization of Porous Materials," Computers and Chemical Engineering journal, Vol. 29, pp. 2134-2143,
2005.

[18] B. Yu, X. He, "Training Radial Basis Function Networks with Differential Evolution," PWASET, Vol. 11, pp. 57$160,2006$.

[19] E.J. Hartman, J.D. Keeler, J.M. Kowalski, "Layered neural networks with Gaussian hidden units as universal approximators," Neural Comput., pp. 210-215, 1990.

[20] J.S.R. Jang, "ANFIS: Adaptive-network-based fuzzy inference systems", IEEE Transactions on Systems, Vol. 23, pp. 665-685, 1993.

[21] J.S.R. Jang, C.T. Sun, E. Mizutani, "A Computational Approach to Learning and Machine Intelligence NeuroFuzzy and Soft Computing," Prentice Hall, US edition, 1997.

[22] S. Shahbudin, A. Hussain, N.M. Tahir, S.A. Samad, "Adaptive-neuro fuzzy inference system for human posture classification using a simplified shock graph", Visual Informatics: Bridging Research and Practice, pp. 585-595, 2009.

[23] T. Culliere, A. Titli, and J. Corrieu, Neuro-fuzzy modeling of nonlinear systems for control purposes," Proc. IEEE Int. Conf.On Fuzzy Systems," pp. 2009-2016, 1995.

\section{Authors' Profiles}

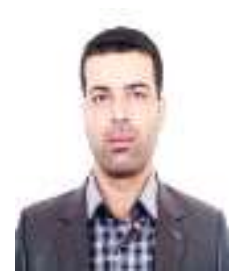

A. Rezaei: Assistance Professor of Electrical Engineering in Kermanshah University of Technology. Abbas Rezaei received the $\mathrm{BSe}, \mathrm{MSe}$ and $\mathrm{PhD}$ in electronics engineering from Razi University, Kermanshah, Iran, in 2005, 2009 and 2013, respectively. His current research interests include computational intelligence,

VLSI and nanotechnology.

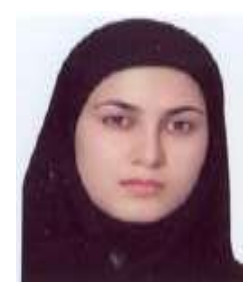

L. Noori: received her B.Sc. and M.Sc. degrees in electronic engineering from Razi University, Kermanshah, Iran in 2005 and 2009 respectively. She recieved her $\mathrm{Ph} . \mathrm{D}$. in electronic engineering at the Shiraz University of Technology. Her research interests focus on artificial microstrip coupler, microstrip filter, neural networks and LNAs.

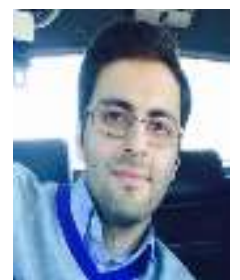

M. Taghipour: MSE degree for Biomedical Engineering in Kermanshah University of Medical Sciences. He was born on Aug 14, 1987 in Neyshabour, Khorasan, IRAN. His research interests are biomedical science and computational intelligence.

How to cite this paper: A. Rezaei, L. Noori, M. Taghipour,"The Use of ANFIS and RBF to Model and Predict the Inhibitory Concentration Values Determined by MTT Assay on Cancer Cell Lines", International Journal of Information Technology and Computer Science(IJITCS), Vol.8, No.4, pp.28-34, 2016. DOI: 10.5815/ijitcs.2016.04.04 